\title{
Nonvacuum Bounces and Quantum Tunneling at Finite Energy
}

\author{
J.-Q. Liang ${ }^{1,2)}$ and H.J.W. Müller-Kirsten ${ }^{1)}$
}

1) Department of Physics, University of Kaiserslautern, P.O. Box 3049, D-67653 Kaiserslautern, Germany

2) CCAST (World Laboratory), P.O. Box 8730, Beijing 100 080;

Institute of Physics; Academia Sinica, Beijing 100 080;

Institute of Theoretical Physics, Shanxi University, Taiyuan, Shanxi 030 006, P.R. China

\begin{abstract}
A bounce-like solution with nonzero energy is used for the explicit calculation of the decay rate of an excited state of the $\phi^{4}$ potential. Three negative eigenvalues are associated with the second variation of the action at the nonvacuum bounce which is different from the case of the vacuum bounce. The imaginary part of the energy results only from the symmetry of the bounce itself, and the additional negative eigenvalues do not present difficulties. In fact, it is shown explicitly that only one negative eigenvalue contributes to the tunneling. The calculated imaginary part of the energy of the excited state is in agreement with that of WKB calculations. The tunneling effects are investigated for high and low energies compared with the barrier height. Finally the Bogomolny-Fateyev relation is established, thus checking our results.
\end{abstract}




\section{Introduction}

The instanton method is by now well known as a powerful tool for dealing with quantum tunneling phenomena. Most of the calculations which have been given in the literature are based on vacuum instantons, namely kinks, with nontrivial topological charge, and these kinks are responsible for quantum tunneling between neighbouring degenerate vacua ${ }^{1}$. It was demonstrated long ago by Coleman and Callen ${ }^{2)}$ that the quantum tunneling process for the decay of a metastable ground state (or "false vacuum") is, however, dominated by a nontopological pseudoparticle configuration named "bounce", which is not a minimum of the action but a saddle point. The second variational derivative of the Euclidean action at the bounce has one negative eigenvalue which leads to the imaginary part of the energy. An explicit calculation of the imaginary part of the energy based on such vacuum bounces has recently been given in ref. [3].

Instanton transitions which relate to the possibility of baryon- and lepton-number violation in electroweak theory have attracted widespread attention ${ }^{4)}$. It has gradually been realised that vacuum instantons and vacuum bounces which prescribe vacuum boundary conditions may not be adequate for the description of tunneling at finite, nonzero energy 5 ). The investigation of quantum tunneling with a new type of instanton-like configurations which are characterized by nonzero energy and satisfy manifestly nonvacuum boundary conditions is therefore of great interest 6 (7)8)9)10)11). In the present paper we investigate the tunneling effect which is dominated by a nonvacuum bounce with nonzero energy which is a case that this is related to a finite temperature process.

The case considered here differs from the vacuum case ${ }^{3)}$ in that the operator associated with the second variation of the Euclidean action at the nonvacuum bounce (which one can also call a periodic bounce) has more than one negative eigenvalue ${ }^{11}$ ). At a first glance this seems to present a difficulty. However, we show explicitly that the imaginary part of the energy results directly from characteristic properties of the bounce itself, namely the antisymmetry of its first time derivative under time reversal. This is in agreement with a general argument given by Coleman ${ }^{9)}$ that if there exist two or more negative eigenvalues 
of the second variational derivative of the action at the bounce only one has to do with tunneling.

In Section 2 we recall from ref. [11] the bounce with nonzero energy for an inverted double-well potential, and the associated equation of small fluctuations about it. In Sections 3 and 4 we present the major part of the procedure for the path-integral calculation of the tunneling process. The tunneling behaviour for the cases a) of energy far below the barrier height, and b) of an energy approaching the barrier height is studied in Section 5 . Finally in Section 6 the Bogomolny-Fateyev relation is established for quantum tunneling through the central barrier of the double-well potential.

\section{Bounces with nonzero energy for the inverted double-well potential}

The Lagrangian for a scalar field $\phi(t)$ in one time and zero space dimensions is

$$
\mathrm{L}=\frac{1}{2}\left[\frac{\mathrm{d} \phi}{\mathrm{dt}}\right]^{2}-\mathrm{V}(\phi)
$$

using unit mass and $\hbar=1$ throughout. The potential $\mathrm{V}(\phi)$ we use is the inverted doublewell potential with a local minimum at $\phi=0$ given by

$$
\mathrm{V}(\phi)=-\frac{\mu^{2}}{2 \mathrm{a}^{2}}\left(\phi^{2}-\mathrm{a}^{2}\right)^{2}+\frac{1}{2} \mu^{2} \mathrm{a}^{2}=\mu^{2} \phi^{2}-\frac{1}{2} \frac{\mu^{2}}{\mathrm{a}^{2}} \phi^{4}
$$

where $\mu$ and a are real parameters. The classical solution $\phi_{\mathrm{C}}$ which minimises the action with Euclidean time $\tau=$ it, satisfies the equation

$$
\frac{1}{2}\left[\frac{\mathrm{d} \phi_{\mathrm{c}}}{\mathrm{d} \tau}\right]^{2}-\mathrm{V}\left(\phi_{\mathrm{c}}(\tau)\right)=-\mathrm{E}_{\mathrm{cl}}
$$

The solution $\phi_{\mathrm{c}}$ is the trajectory of the classical pseudoparticle. The integration constant $\mathrm{E}_{\mathrm{cl}} \geq 0$ can be regarded as the energy of the classical particle. If one wishes, one can look at the solution as describing the motion of a real particle with energy $-\mathrm{E}_{\mathrm{cl}}$ in the potential $-\mathrm{V}(\phi)$. It is convenient to set 


$$
\mathrm{E}_{\mathrm{cl}}=\frac{1}{2} \mathrm{a}^{2} \mu^{2} \mathrm{u}^{2}, \quad \mathrm{u}=\frac{1-\mathrm{k}^{2}}{1+\mathrm{k}^{2}}, \quad 0 \leq \mathrm{k} \leq 1
$$

This substitution defines the parameter $\mathrm{k}$ which varies from 0 to 1 as $\mathrm{E}_{\mathrm{cl}}$ varies between its extreme values, i.e.

$$
-\frac{1}{2} \mu^{2} \mathrm{a}^{2} \leq-\mathrm{E}_{\mathrm{cl}} \leq 0
$$

We demand that $\phi_{c}(\tau)$ be periodic with period $I$ so that

$$
\phi_{c}(\tau)=\phi_{c}(\tau+I)
$$

The solution of (2.2) is then given by

$$
\phi_{\mathrm{c}}(\tau)=\mathrm{s}_{+}(\mathbf{k}) \mathrm{dn}\left[\beta(\mathbf{k})\left(\tau+\tau_{0}\right) \mid \gamma\right]
$$

where dn $u$ (like $\operatorname{sn} u, c n u$ ) denotes a Jacobian elliptic function, $\tau_{0}$, an integration constant, defines the position of the bounce, and $\gamma$ is the modulus of the elliptic functions which is related to $\mathrm{k}$ by

$$
\gamma^{2}=\frac{4 k}{(1+k)^{2}}, \quad \gamma^{\prime 2}=1-\gamma^{2}=\left[\frac{1-k}{1+k}\right]^{2}=\frac{1-u^{\prime}}{1+u^{\top}}, \quad u^{\prime 2}=1-u^{2}
$$

The other $\mathbf{k}$-dependent parameters in (2.3) are given by

$$
\beta(\mathrm{k})=\frac{\mu}{\mathrm{a}} \mathrm{s}_{+}(\mathrm{k}), \quad \mathrm{s}_{+}(\mathrm{k})=\frac{\mathrm{a}(1+\mathrm{k})}{\sqrt{1+\mathrm{k}^{2}}}=\mathrm{a}\left[\frac{2}{1+\gamma^{2}}\right]^{\frac{1}{2}} \equiv \mathrm{s}_{+}(\gamma)
$$

The Jacobian elliptic function dn $[\beta(\mathbf{k}) \tau \mid \gamma]$ has period

$$
\beta(\mathrm{k}) \mathrm{T}=\mathrm{n} .2 \mathcal{K}(\gamma), \quad \mathrm{n}=1, \ldots
$$


where $\mathcal{X}(\gamma)$ is the elliptic quarter period or complete elliptic integral of the first kind. The pseudoparticle oscillaters from turning point $\tilde{a}$ to $\tilde{a}^{\prime}$ and back in the barrier as shown in Fig. 1. Setting $T \equiv 2 \mathrm{~T}$ and taking $\mathrm{n}=1$, we have

$$
\beta(\mathbf{k}) \mathrm{T}=\chi(\gamma)
$$

with $\mathrm{T}$ as half the period of the motion of the pseudoparticle as indicated in Fig. 1. As the energy tends to zero with $k \rightarrow 1$, the solution (2.3) reduces to the usual vacuum bounce, i.e.

$$
\phi_{C}(\tau) \rightarrow a \sqrt{2} \operatorname{sech}\left[\mu \sqrt{2}\left(\tau+\tau_{0}\right)\right]
$$

For the sake of a better distinction we dub the new solution of eq. (2.3) a "nonvacuum bounce" or periodic bounce. On the other hand, as the energy approaches the top of the barrier, i.e. $\mathrm{E}_{\mathrm{cl}}=\frac{1}{2} \mu^{2} \mathrm{a}^{2}$, with $\mathrm{k} \rightarrow 0$, the solution becomes the trivial configuration $\phi_{\mathrm{C}}=\mathbf{a}$ since $^{12)} \operatorname{dn}[u \mid \gamma]=1$ for $\gamma=0$. This trivial solution is called a sphaleron ${ }^{10) 11)}$. The nonvacuum bounce thus interpolates between the vacuum bounce and this sphaleron.

The small fluctuation equation about the classical solution $\phi_{\mathrm{c}}(\tau)$ is

$$
\hat{\mathrm{M}} \psi=\omega^{2} \psi
$$

with

$$
\hat{\mathrm{M}}=-\frac{\mathrm{d}^{2}}{\mathrm{~d} \tau^{2}}+\mu^{2}\left[2-\frac{6}{\mathrm{a}^{2}} \phi_{\mathrm{c}}^{2}\right]
$$

and can be reexpressed as the Lamé equation

$$
\frac{\mathrm{d}^{2} \psi}{\mathrm{dz}}+\left\{\omega^{2}-6 \gamma^{2} \mathrm{sn}^{2}[\mathrm{z} \mid \gamma]\right\} \psi=0
$$

where $\mathbf{z}=\beta(\mathbf{k}) \tau$. It will be seen later that $\hat{\mathbf{M}}$ is simply the second variational derivative of the Euclidean action at the bounce $\phi_{c}$. The discrete eigenmodes $\psi_{1}, \psi_{2}, \ldots, \psi_{5}$ of $(2.6)$ are given in ref. [11] as 


$$
\begin{aligned}
& \text { sn }[z \mid \gamma] \text { cn }[z \mid \gamma], \text { sn }[z \mid \gamma] \operatorname{dn}[z \mid \gamma] \\
& \text { cn }[z \mid \gamma] \text { dn }[z \mid \gamma]
\end{aligned}
$$

and

$$
\operatorname{sn}^{2}[\mathrm{z} \mid \gamma]-\frac{1}{3 \gamma^{2}}\left[1+\gamma^{2} \pm \sqrt{1-\gamma^{2} \gamma^{\prime 2}}\right]
$$

with eigenvalues (expressed in terms of $\mathrm{k}$ ) $\omega^{2} \rightarrow \omega_{1}^{2}, \omega_{2}^{2}, \ldots, \omega_{5}^{2}$ given by

$$
0,-\frac{3 \mu^{2}(1-k)^{2}}{1+k^{2}},-\frac{3 \mu^{2}(1+k)^{2}}{1+k^{2}}
$$

and

$$
-2 \mu^{2} \mp 2 \mu^{2} \frac{\sqrt{1+14 \mathrm{k}^{2}+\mathrm{k}^{4}}}{1+\mathrm{k}^{2}}
$$

respectively. For $k \in(0,1)$ there are three negative eigenvalues, their respective eigenmodes having periods $T=4 \mathrm{n} X(\gamma) / \beta(\mathrm{k})$. The topological charge of $\phi_{\mathrm{c}}$ is zero (as a consequence of the periodicity of $\phi_{C}$ ) and therefore $\phi_{c}$ is an unstable, nontopological pseudoparticle configuration.

\section{Quantum tunneling from an excited state}

We let $|\mathrm{E}\rangle$ be an eigenstate of the Hamiltonian $\hat{\mathrm{H}}$ with energy $\mathrm{E}$. Due to tunneling and escape to infinity $\mathrm{E}$ becomes a complex number in the case under discussion, with the imaginary part characterising the rate of decay of the state. The quantity we wish to calculate is precisely this imaginary part of the energy $\mathrm{E}$.

To begin with we consider the transition amplitude from the state $\mathbf{E}$ to itself due to quantum tunneling in Euclidean time 2T, i.e.

$$
A=\left\langle E\left|e^{-2 \hat{H} T}\right| E\right\rangle=e^{-2 E T},\langle E \mid E\rangle=1
$$


The amplitude can be calculated with the help of the path-integral method. We rewrite it

$$
\mathrm{A}=\int \psi_{\mathrm{E}}^{*}\left(\phi_{\mathrm{f}}\right) \psi_{\mathrm{E}}\left(\phi_{\mathrm{i}}\right) \mathrm{K}\left(\phi_{\mathrm{f}}, \tau_{\mathrm{f}} ; \phi_{\mathrm{i}}, \tau_{\mathrm{i}}\right) \mathrm{d} \phi_{\mathrm{f}} \mathrm{d} \phi_{\mathrm{i}}
$$

with $\phi_{\mathrm{f}}=\phi\left(\tau_{\mathrm{f}}\right), \phi_{\mathrm{i}}=\phi\left(\tau_{\mathrm{i}}\right)$ and $\tau_{\mathrm{f}}-\tau_{\mathrm{i}}=2 \mathrm{~T}$

Thus $\phi_{\mathrm{i}}$ and $\phi_{\mathrm{f}}$ denote the endpoints of the bounce motion which tend to the turning point a with $\phi\left(\tau_{\mathrm{i}}\right) \equiv \phi_{\mathrm{i}}=\phi\left(\tau_{\mathrm{f}}\right) \equiv \phi_{\mathrm{f}} \rightarrow \mathrm{a}$. The Feynman propagator from $\phi_{\mathrm{i}}$ to $\phi_{\mathrm{f}}$ resulting from the bounce-motion is defined by

$$
\mathrm{K}\left(\phi_{\mathbf{f}}, \tau_{\mathbf{f}} ; \phi_{\mathrm{i}}, \tau_{\mathbf{i}}\right)=\underbrace{\phi_{\mathrm{f}}}_{\oint_{\mathrm{i}}} \mathcal{D}\{\phi\} \exp (-\mathrm{S})
$$

where

$$
\mathrm{S}=\int_{\tau_{\mathrm{i}}}^{\tau_{\mathbf{f}}}\left[\frac{1}{2}\left[\frac{\mathrm{d} \phi}{\mathrm{d} \tau}\right]^{2}+\mathrm{V}(\phi)\right] \mathrm{d} \tau
$$

is the classical action. Here, i.e. in (3.2)

$$
\psi_{\mathrm{E}}\left(\phi_{\mathrm{f}}\right) \equiv\left\langle\phi_{\mathrm{f}} \mid \mathrm{E}\right\rangle \text { and } \psi_{\mathrm{E}}\left(\phi_{\mathrm{i}}\right) \equiv\left\langle\phi_{\mathrm{i}} \mid \mathrm{E}\right\rangle
$$

are wave functions to be specified later.

\section{The imaginary part of the energy}

We derive the imaginary part of the energy by considering the amplitude $A$ as the sum of contributions from any number of bounces. The first contribution $A^{(0)}$ in the sum is that of no bounces, and so of no tunneling. In this approximation the shoulders of the inverted double-well potential are infinitely high and the eigenenergy $E$ of (3.1) is the energy of that excited state of the harmonic oscillator with minimum at $\phi=0$, which is closest to the classical energy $\mathrm{E}_{\mathrm{cl}}$. Remembering that we assume unit mass of the pseudoparticle and $\hbar \equiv 1$ this implies 


$$
\mathrm{A}^{(0)}=\mathrm{e}^{-2 \mathrm{E}} \mathrm{cl}^{\mathrm{T}}
$$

with $\mathrm{E}_{\mathrm{cl}} \simeq(2 \mathrm{n}+1) \frac{\mu}{\sqrt{2}}, \mathrm{n}=0,1, \ldots$

Next we consider the various bounce contributions.

\section{(A) The one bounce contribution}

The Feynman propagator or kernel $\mathrm{K}$ defined by (3.3) can be evaluated with the standard path-integral method. Considering fluctuations about the bounce $\phi_{c}(\tau)$ we set

$$
\phi(\tau)=\phi_{c}(\tau)+\chi(\tau)
$$

where $\chi(\tau)$ denotes the small deviation of $\phi$ from the classical trajectory with end points held fixed. Thus necessary boundary conditions for $\chi(\tau)$ are

$$
\chi\left(\tau_{\mathrm{f}}\right)=\chi\left(\tau_{\mathrm{j}}\right)=0
$$

Substituting (4.1) for $\phi(\tau)$ in eq. (3.3), we obtain

$$
\mathrm{K}=\exp \left[-\mathrm{S}_{\mathrm{c}}(\phi)\right] . \mathbf{I}
$$

where $I$ is the functional integral

$$
\mathrm{I}=\int_{\chi\left(\tau_{\mathrm{i}}\right)=0}^{\chi\left(\tau_{\mathrm{f}}\right)=0} \mathcal{D}\{\chi\} \exp [-\delta \mathrm{S}]
$$

Here

$$
\begin{aligned}
\mathrm{S}_{\mathrm{c}}(\phi) & =\int_{\tau_{\mathrm{i}}}^{\tau_{\mathrm{f}} \mathrm{d} \tau}\left[\frac{1}{2}\left[\frac{\mathrm{d} \phi_{\mathrm{c}}}{\mathrm{d} \tau}\right]^{2}+\mu^{2} \phi_{\mathrm{c}}^{2}-\frac{1}{2} \frac{\mu^{2}}{\mathrm{a}^{2}} \phi_{\mathrm{c}}^{4}\right] \\
& =\int_{\tau_{\mathrm{i}}}^{\tau_{\mathrm{f}}} \mathrm{d} \tau\left[\left[\frac{\mathrm{d} \phi_{\mathrm{c}}}{\mathrm{d} \tau}\right]^{2}+\mathrm{E}_{\mathrm{cl}}\right]
\end{aligned}
$$


and up to terms of $\mathrm{O}\left(\chi^{2}\right)$ (for weak coupling) $\delta \mathrm{S}$ is given by

$$
\begin{aligned}
\delta \mathrm{S}= & \frac{1}{2} \int_{\tau_{\mathbf{i}}}^{\tau_{\mathrm{f}} \mathrm{d} \tau}\left[-\chi \frac{\mathrm{d}^{2} \chi}{\mathrm{d} \tau^{2}}+2 \chi\left[\mu^{2}-\frac{3 \mu^{2}}{\mathrm{a}^{2}} \phi_{\mathrm{c}}^{2}\right] \chi\right] \\
& \equiv \frac{1}{2} \int_{\tau_{\mathbf{i}}}^{\tau_{\mathrm{f}}} \mathrm{d} \tau \hat{\mathbf{M}} \chi
\end{aligned}
$$

where $\hat{\mathrm{M}}$ is the operator defined by (2.5). Chosing the bounce position $\tau_{0}=0$ and inserting into (4.4) the derivative of $\phi_{c}(\tau)$, i.e.

$$
\frac{\mathrm{d} \phi_{\mathrm{c}}}{\mathrm{d} \tau}=-\mathrm{s}_{+}(\mathrm{k}) \beta(\mathrm{k}) \gamma^{2} \operatorname{sn}[\beta(\mathrm{k}) \tau \mid \gamma] \operatorname{cn}[\beta(\mathbf{k}) \tau \mid \gamma]
$$

we can evaluate (4.4) with the help of tables of integrals ${ }^{12)}$ and find with $\tau_{\mathrm{i}}=-\mathrm{T}$, $\tau_{\mathrm{f}}=+\mathrm{T}$, and $\mathrm{T}=\mathrm{K}(\gamma) / \beta(\mathrm{k})$

$$
\mathrm{S}_{\mathrm{c}}(\phi)=\mathrm{W}\left(\phi\left(\tau_{\mathrm{f}}\right), \phi\left(\tau_{\mathrm{i}}\right), \mathrm{E}_{\mathrm{cl}}\right)+2 \mathrm{E}_{\mathrm{cl}} \mathrm{T}
$$

where for $\phi\left(\tau_{\mathbf{j}}\right) \rightarrow \tilde{\mathrm{a}}, \phi\left(\tau_{\mathbf{f}}\right) \rightarrow \tilde{\mathrm{a}}^{\prime}($ cf. Fig. 1)

$$
\begin{aligned}
& \mathrm{W}\left(\phi\left(\tau_{\mathrm{f}}\right), \phi\left(\tau_{\mathrm{i}}\right), \mathrm{E}_{\mathrm{cl}}\right) \\
& \rightarrow \gamma^{4} \mathrm{~s}_{+}^{2}(\mathrm{k}) \beta(\mathrm{k}){ }_{-\mathcal{X}(\gamma)}^{\mathcal{X}(\gamma)} \mathrm{du} \mathrm{sn}^{2}[\mathrm{u} \mid \gamma] \mathrm{cn}^{2}[\mathrm{u} \mid \gamma] \\
& \quad=\frac{2}{3} \mathrm{~s}_{+}^{2}(\mathrm{k}) \beta(\mathrm{k})\left[\left(2-\gamma^{2}\right) \mathrm{E}(\mathcal{X}(\gamma))-2{\gamma^{\prime}}^{2} \chi(\gamma)\right]
\end{aligned}
$$

Here $\mathrm{E}(\mathcal{X}(\gamma))$ is the complete elliptic integral of the second kind.

The traditional way of deriving the imaginary part of the energy as described by Coleman and Callan ${ }^{2)}$ is to expand $\chi$ in terms of the eigenfunctions of $\hat{\mathbf{M}}$, i.e. those of (2.5). The 
evaluation of $I$ then leads to a divergent Gaussian integral as a result of an associated negative eigenvalue. By continuation into the complex plane the integral acquires an imaginary part. One therefore needs one and only one negative eigenmode. In our case, however, there are three negative eigenmodes. Nonetheless, this does not lead to a difficulty here, since - as will be seen - the boundary condition (4.2) selects precisely one of these modes, namely the third of $(2.7 \mathrm{a}), \operatorname{cn}[z \mid \gamma] \operatorname{dn}[z \mid \gamma]$, which together with the zero mode $\operatorname{sn}[z \mid \gamma] \operatorname{cn}[z \mid \gamma]$ contributes to the path-integral.

Instead of expanding $\chi(\tau)$ in terms of the eigenmodes of $\hat{\mathrm{M}}$, we resort to an alternative method in evaluating the functional integral I for our purposes. We perform the transformation to $\mathrm{y}(\tau)$ given by

$$
\chi(\tau)=\mathrm{y}(\tau)+\mathrm{N}(\tau) \int_{\tau_{\mathrm{i}}}^{\tau} \frac{\dot{\mathrm{N}}\left(\tau^{\prime}\right)}{\mathrm{N}^{2}\left(\tau^{\prime}\right)} \mathrm{y}\left(\tau^{\prime}\right) \mathrm{d} \tau^{\prime}
$$

where

$$
\mathrm{N}(\tau)=\frac{\mathrm{d} \phi_{\mathrm{C}}(\tau)}{\mathrm{d} \tau}
$$

is the unnormalised zero mode of the small fluctuation equation (2.5). The evaluation of the path-integral I of eq. (4.3) can then be carried out by direct integration ${ }^{8) 13) 14}$ ). The result is (as we show in Appendix B)

$$
\mathrm{I}=\left[\frac{1}{2 \pi}\right]^{\frac{1}{2}}\left[\frac{1}{\mathrm{~N}\left(\tau_{\mathrm{f}}\right) \mathrm{N}\left(\tau_{\mathrm{i}}\right)}\right]^{\frac{1}{2}}\left[\int_{\tau_{\mathrm{i}}}^{\tau_{\mathrm{f}}} \frac{\mathrm{d} \tau}{\mathrm{N}^{2}(\tau)}\right]^{-\frac{1}{2}}
$$

with $\tau_{\mathrm{i}}=-\mathrm{T}, \tau_{\mathrm{f}}=\mathrm{T}$.

We observe that the bounce solution $\phi_{C}$ is symmetric under time-reversal (since $\mathrm{dn}(\mathrm{u})$ $=\mathrm{dn}(-\mathrm{u}))$ whereas its derivative $\mathrm{N}(\tau)=\mathrm{d} \phi_{\mathrm{c}} / \mathrm{d} \tau$ is antisymmetric (since $\operatorname{sn}(-\mathrm{u})=-\operatorname{sn}(\mathrm{u})$ 
and $\mathrm{cn}(-\mathrm{u})=\mathrm{cn}(\mathrm{u}))$. The square root of $\mathrm{N}(\mathrm{T}) \mathrm{N}(-\mathrm{T})$ therefore implies that $\mathrm{I}$ is imaginary. In Appendix A we show that in fact only one of the three eigenmodes with negative eigenvalue actually contributes to the quantum tunneling.

The propagator (4.3a) for motion from the turning point $\phi=\tilde{a}$ and load to $\tilde{a}$ (i.e. $\tau= \pm \mathrm{T}$ ) is divergent because of the vanishing velocity at turning points (i.e. $\operatorname{cn}( \pm \mathcal{X})=0$ in $(4.6)$ ). This is unlike the case of vacuum bounces which can reach the turning points only asymptotically.

The transition amplitude, of course, has to be finite, and hence the singularity of the propagator has to be smoothed out by the endpoint integrations of $d \phi_{i}$ and $d \phi_{f}$. To this end we use the following relations established in Appendix C

$$
I=\left[\frac{1}{2 \pi}\right]^{\frac{1}{2}}\left[\frac{N\left(\phi_{f}\right)}{N\left(\phi_{i}\right)}\right]^{\frac{1}{2}}\left[\frac{\partial^{2} S_{c}\left(\phi_{f}, \phi_{i} ; T\right)}{\partial \phi_{f}^{2}}\right]^{\frac{1}{2}} \Delta\left(\phi_{f}, \phi_{i}\right)
$$

where

$$
\frac{\partial^{2} \mathrm{~S}_{\mathrm{c}}\left(\phi_{\mathrm{f}}, \phi_{\mathrm{i}} ; \mathrm{T}\right)}{\partial \phi_{\mathrm{f}}^{2}}=\frac{1}{\mathrm{~N}\left(\phi_{\mathrm{f}}\right)}\left[\frac{\partial^{2} \phi_{\mathrm{f}}}{\partial \tau^{2}}+\frac{1}{\mathrm{~N}\left(\phi_{\mathrm{f}}\right) \int_{\tau_{\mathrm{i}}{ }_{\mathrm{f}} \mathrm{d} \tau}^{\mathrm{N}^{2}(\tau)}}\right]
$$

and

$$
\Delta\left(\phi_{\mathrm{f}}, \phi_{\mathrm{i}}\right)=\left[\frac{\partial^{2} \mathrm{~S}_{\mathrm{c}}\left(\phi_{\mathrm{f}}, \phi_{\mathrm{i}} ; \mathrm{T}\right)}{\partial \phi_{\mathrm{f}}^{2}} \mathrm{~N}^{2}\left(\phi_{\mathrm{f}}\right) \int_{\tau_{\mathrm{i}}}^{\tau_{\mathrm{f}}} \frac{\mathrm{d} \tau}{\mathrm{N}^{2}(\tau)}\right]^{-\frac{1}{2}}
$$

with

$$
\Delta \equiv \lim _{\substack{\phi_{\mathrm{f}} \rightarrow \tilde{\mathrm{a}} \\ \phi_{\mathrm{i}} \rightarrow \tilde{\mathrm{a}}}} \Delta\left(\phi_{\mathrm{f}}, \phi_{\mathrm{i}}\right)=-\mathrm{i}
$$

Eq. (4.11a) follows, of course, from (4.10) and (4.11b) and (4.12a). 
We evaluate the amplitude $A$ of (3.2) by replacing the wave functions $\psi_{E}\left(\phi_{\mathrm{f}}\right), \psi_{\mathrm{E}}\left(\phi_{\mathrm{i}}\right)$ by their leading WKB approximations, and by expanding the action $\mathrm{S}$ in (4.12) (which is a function of the endpoints $\phi_{i}$ and $\left.\phi_{f}\right)$ in powers of $\phi_{f}-\phi_{c}(T)$ up to the second power for the Gaussian or one-loop approximation. Thus we write

$$
\begin{aligned}
\mathrm{S}_{\mathrm{c}}(\phi) & \equiv \mathrm{S}_{\mathrm{c}}\left(\phi_{\mathrm{f}}, \phi_{\mathrm{i}}, \mathrm{T}\right) \\
& =\mathrm{S}_{\mathrm{c}}(\phi(\mathrm{T}), \phi(-\mathrm{T}), \mathrm{T}) \\
& +\frac{1}{2} \frac{\partial^{2} \mathrm{~S}}{\partial \phi(\mathrm{T})^{2}}\left(\phi_{\mathrm{f}}-\phi(\mathrm{T})\right)^{2}+\ldots
\end{aligned}
$$

We define

$$
\begin{aligned}
& \tilde{\mathrm{a}} \equiv \phi(-\mathrm{T})=\phi(\mathrm{T}) \\
& \tilde{\mathrm{a}}^{\prime} \equiv \phi(0)
\end{aligned}
$$

as turning points. Inserting (2.3) here we obtain

$$
\tilde{\mathrm{a}}=\frac{\mathrm{a}(1-\mathrm{k})}{\sqrt{1+\mathrm{k}^{2}}}, \quad \tilde{\mathrm{a}}^{\prime}=\frac{\mathrm{a}(1+\mathrm{k})}{\sqrt{1+\mathrm{k}^{2}}}
$$

These turning points are shown in Fig. 1. The WKB approximations of the wave functions are given by

$$
\psi_{\mathrm{E}}\left(\phi_{\mathrm{f}}\right)=\mathrm{C} \frac{\mathrm{e}^{-\int_{\tilde{\mathrm{a}}}^{\phi_{\mathrm{f}}} \dot{\phi} \mathrm{d} \phi}}{\sqrt{\dot{\phi}_{\mathrm{f}}}}, \quad \psi_{\mathrm{E}}\left(\phi_{\mathrm{i}}\right)=\mathrm{C} \frac{\mathrm{e}^{-\int_{\tilde{\mathrm{a}}}^{\phi_{\mathrm{i}}} \dot{\phi} \mathrm{d} \phi}}{\sqrt{\dot{\phi}_{\mathrm{i}}}}
$$

The normalisation constant $\mathrm{C}$ is defined, as usual, by 


$$
\mathrm{C}=\left[\frac{1 / 2}{\int_{-\tilde{\mathrm{a}}} \frac{\mathrm{d} \phi}{\sqrt{2(\mathrm{E}-V(\phi))}}}\right]^{\frac{1}{2}}
$$

where the integration extends from turning point to turning point across the nontunnelng domain (i.e. the region of the harmonic oscillator approximation). Evaluating $\mathrm{C}$ one obtains

$$
C=\left[\frac{\mu(1+k)}{4 \sqrt{1+k^{2}} x\left(\gamma^{\prime}\right)}\right]^{\frac{1}{2}}
$$

(using integrals given in ref. 12) and the relation $\mathrm{F}\left[\frac{\pi}{2}, \gamma^{\prime}\right]=\mathcal{X}\left(\gamma^{\prime}\right)$ ). The endpoint integrations in eq. (3.2) can now be carried out for the one-bounce contribution $\mathrm{A}^{(1)}$. We have for $\tau_{\mathrm{f}} \rightarrow \mathrm{T}, \tau_{\mathrm{i}} \rightarrow-\mathrm{T}$, using (4.3), (4.11), (4.12) and (4.15) and writing $\mathrm{d} \phi_{\mathrm{i}}=\dot{\phi}_{\mathrm{i}} \mathrm{d} \tau$,

$$
\begin{aligned}
\mathrm{A}^{(1)}= & \int \psi_{\mathrm{E}}^{*}\left(\phi_{\mathrm{f}}\right) \psi_{\mathrm{E}}\left(\phi_{\mathrm{i}}\right) \mathrm{K}\left(\phi_{\mathrm{f}}, \tau_{\mathrm{f}} \phi_{\mathrm{i}}, \tau_{\mathrm{i}}\right) \mathrm{d} \phi_{\mathrm{f}} \mathrm{d} \phi_{\mathrm{i}} \\
= & \int \psi_{\mathrm{E}}^{*}\left(\phi_{\mathrm{f}}\right) \psi_{\mathrm{E}}\left(\phi_{\mathrm{i}}\right) \mathrm{I} \exp \left[-\mathrm{S}_{\mathrm{c}}(\phi)\right] \mathrm{d} \phi_{\mathrm{f}} \mathrm{d} \phi_{\mathrm{i}} \\
\simeq & \frac{\mathrm{C}^{2}}{\sqrt{2 \pi}} \int \frac{\mathrm{d} \phi_{\mathrm{f}} \mathrm{d} \phi_{\mathrm{i}} \Delta}{\phi_{\mathrm{i}}} \sqrt{\frac{\partial^{2} \mathrm{~S}_{\mathrm{c}}}{\partial \phi(\mathrm{T})^{2}}} \exp \left[-\mathrm{S}_{\mathrm{c}}(\phi)\right] \\
\simeq & \frac{\mathrm{C}^{2}}{\sqrt{2 \pi}} \int_{-\mathrm{T}}^{\mathrm{T}} \mathrm{d} \tau \int \Delta \sqrt{\frac{\partial^{2} \mathrm{~S}_{\mathrm{c}}}{\partial \phi(T)^{2}}} \mathrm{~d} \phi_{\mathrm{f}} \mathrm{e}^{-\mathrm{S}_{\mathrm{c}}[\phi(\mathrm{T}), \phi(-\mathrm{T}), \mathrm{T}]} \\
& \cdot \exp \left[-\frac{1}{2} \frac{\partial^{2} \mathrm{~S}_{\mathrm{c}}}{\left.\partial \phi(\mathrm{T})^{2}\left(\phi_{\mathrm{f}}-\phi(\mathrm{T})\right)^{2}\right]}\right. \\
\simeq & -\mathrm{i} 2 \mathrm{~T} \cdot \mathrm{C}^{2} \mathrm{e}^{-\mathrm{W}} \cdot \mathrm{e}^{-2 \mathrm{E}_{\mathrm{cl}} \mathrm{T}}
\end{aligned}
$$

where in the last step we used (4.12b) and (4.7). 
Inserting (4.17) this becomes

$$
\mathrm{A}^{(1)}=(-\mathrm{i}) \cdot 2 \mathrm{~T} \cdot \mathrm{e}^{-\mathrm{W}} \mathrm{e}^{-2 \mathrm{E}} \mathrm{cl} \frac{\mu(1+\mathrm{k})}{4 \sqrt{1+\mathrm{k}^{2}} \mathfrak{X}\left(\gamma^{\prime}\right)}
$$

\section{(B) Summing over an infinite number of bounces}

The path integral implies a sum over all possible paths. The one-bounce contribution is that of the classical configuration (2.3) with period $T=\mathrm{n} \cdot 2 \chi(\gamma) / \beta(\mathrm{k})$ (eq. (2.4)) with $\mathrm{n}=1$. For $\mathrm{n}=2$ we have (since $T=2 \mathrm{~T}$ ) $\beta(\mathrm{k}) \mathrm{T}=2 \chi(\gamma)$, and so there are two bounces moving from $-\mathrm{T}$ to $+\mathrm{T}$ with "positions" $\tau_{0}= \pm \mathcal{X}(\gamma) / \beta(\mathrm{k})$ as shown in Fig. 1. The contribution $\mathrm{A}^{(2)}$ to the transition amplitude arising from two bounces can be calculated in analogy to the contribution of an instanton-antiinstanton pair to the leading instanton contribution as discussed in ref. [1]. Thus

$$
\begin{aligned}
\mathrm{A}^{(2)} & =\int_{-\mathrm{T}}^{\mathrm{T}} \mathrm{d} \tau \int_{-\mathrm{T}}^{\tau} \mathrm{d} \tau_{1}(-\mathrm{i})^{2} \mathrm{e}^{-2 \mathrm{~W}} \mathrm{e}^{-2 \mathrm{E}} \mathrm{cl} \mathrm{T}^{\mathrm{T}}\left[\frac{\mu(1+\mathrm{k})}{4 \sqrt{1+\mathrm{k}^{2}} \chi\left(\gamma^{\prime}\right)}\right]^{2} \\
& =(-\mathrm{i})^{2} \frac{(2 \mathrm{~T})^{2}}{2 !}\left[\frac{\mu(1+\mathrm{k})}{4 \sqrt{1+\mathrm{k}^{2}} \chi\left(\gamma^{\prime}\right)}\right]^{2} \mathrm{e}^{-2 \mathrm{~W}} \cdot \mathrm{e}^{-2 \mathrm{E} \mathrm{cl}^{\mathrm{T}}}
\end{aligned}
$$

The generalisation to $\mathrm{n}$ bounces is now straightforward. We have

$$
\mathrm{A}^{(\mathrm{n})}=(-\mathrm{i})^{\mathrm{n}} \frac{(2 \mathrm{~T})^{\mathrm{n}}}{2 !}\left[\frac{\mu(1+\mathrm{k})}{4 \sqrt{1+\mathrm{k}^{2}} \chi\left(\gamma^{\prime}\right)}\right]^{\mathrm{n}} \mathrm{e}^{-\mathrm{nW}} \cdot \mathrm{e}^{-2 \mathrm{E} c \mathrm{cl}^{\mathrm{T}}}
$$

The total transition amplitude $\mathrm{A}$ which results from quantum tunneling dominated by bounces is obtained by summation, i.e.

$$
A=\underset{n}{\Sigma} A^{(n)}=e^{-2 E_{c l} T} \exp \left\{-i \cdot 2 T \frac{\mu(1+k)}{4 \sqrt{1+k^{2}} \chi\left(\gamma^{\prime}\right)} e^{-W}\right\}
$$

Comparing this expression with (3.1) we see that the imaginary part of the energy is given by 


$$
\operatorname{Im} E=\frac{\mu(1+k)}{4 \sqrt{1+k^{2}} \chi\left(\gamma^{\prime}\right)} e^{-W}
$$

where $\mathrm{W}$ is given by $(4.8)$, i.e.

$$
\begin{aligned}
\operatorname{Im} E & =\frac{\mu(1+\mathrm{k})}{4 \sqrt{1+\mathrm{k}^{2}} \chi\left(\gamma^{\prime}\right)} \exp \left[-\frac{2^{\frac{5}{2}}}{3} \frac{\mu \mathrm{a}^{2}}{\left(1+\gamma^{\prime 2}\right)^{3 / 2}}\right] \\
& \left.\cdot\left\{\left(2-\gamma^{2}\right) \mathrm{E}(\mathcal{X}(\gamma))-2{\gamma^{\prime}}^{2} \mathcal{X}(\gamma)\right\}\right]
\end{aligned}
$$

This expression is nothing but a WKB formula which is similar to the well-known levelsplitting formula ${ }^{8) 15) 16)}$ for a double-well potential.

\section{Low and high energy limits}

It is interesting to investigate the low and high energy limits of eq. (4.23). By "low" and "high" energies we mean energies far below or near the barrier maximum respectively.

(A) For the energy far below the barrier maximum $E_{c l}<<\frac{1}{2} a^{2} \mu^{2}$ and therefore $k^{2} \rightarrow 1$ or $\gamma^{2} \rightarrow 1$. For $\gamma \rightarrow 1$ the quarter period $\chi(\gamma) \rightarrow \infty$ and $^{12)} \mathrm{E}(X=\infty)=\mathrm{E}(\mathrm{k}=1)$. The expansions of $\mathrm{E}(\gamma)$ and $\mathcal{X}(\gamma)$ in this domain (or correspondingly for ${\gamma^{\prime}}^{2} \rightarrow 0$ ) are ${ }^{12}$ )

$$
\begin{aligned}
\mathrm{E}(\gamma) & =1+\frac{1}{2}\left\{\ln \left[\frac{4}{\gamma^{\prime}}\right]-\frac{1}{2}\right\}{\gamma^{\prime}}^{2} \\
& +\frac{3}{16}\left\{\ln \left[\frac{4}{\gamma^{\prime}}\right]-\frac{13}{12}\right\} \gamma^{\prime 4}+\ldots
\end{aligned}
$$

and

$$
\chi(\gamma)=\ln \left[\frac{4}{\gamma^{\prime}}\right]+\frac{1}{4}\left[\ln \left[\frac{4}{\gamma^{\prime}}\right]-1\right] \gamma^{\prime 2}+\ldots
$$

The parameters $\mathbf{k}$ or $\gamma$ parametrize the energy $\mathrm{E}_{\mathrm{cl}}$. The latter is quantized either by reference to the harmonic oscillator at the central well (with mass and $\hbar=1$ ) or by using the Bohr-Sommerfeld condition 


$$
\int_{-\tilde{a}}^{\tilde{a}} \frac{d \phi}{d t} d \phi=\left[n+\frac{1}{2}\right] \pi
$$

and keeping only terms up to those of $o\left({\gamma^{\prime}}^{2}\right)$. In either case one finds

$$
\mathrm{E}_{\mathrm{cl}}=\left[\mathrm{n}+\frac{1}{2}\right] \omega, \omega=\mu \sqrt{2}
$$

and $\mathrm{n}=0,1, \ldots$

Inserting $\mathrm{E}(\gamma)$ and $\mathcal{X}(\gamma)$ into the exponent of (4.23) we obtain for $W$

$$
\mathrm{W}=\frac{2^{\frac{5}{2}} \mu \mathrm{a}}{3}\left[1-\frac{3}{4}{\gamma^{\prime}}^{2}-\frac{3}{2}{\gamma^{\prime}}^{2} \ln \left[\frac{4}{\gamma^{\prime}}\right]\right]
$$

Setting

$$
\mathrm{g}^{2} \equiv \frac{1}{\mu \mathrm{a}^{2}}
$$

(a dimensionless coupling constant) then, since

$$
{\gamma^{\prime}}^{2}=\frac{1-\mathrm{u}^{\prime}}{1+\mathrm{u}^{\prime}} \simeq \frac{\mathrm{u}^{2}}{4}
$$

we obtain in the domain of weak coupling, i.e. for $\mathrm{g}^{2}<<1$,

$$
W=\frac{4 \sqrt{2}}{3 g^{2}}\left[1-\frac{3}{16} u^{2}-\frac{3}{8} u^{2} \ln \left[\frac{8}{u}\right]\right]
$$

Since $\mathrm{E}_{\mathrm{cl}}=\frac{1}{2} \mathrm{a}^{2} \mu^{2} \mathrm{u}^{2}$, we obtain with (5.3)

$$
\mathrm{u}^{2}=2 \sqrt{2}\left[\mathrm{n}+\frac{1}{2}\right] \mathrm{g}^{2}
$$


Inserting this into (5.5) implies

$$
\mathrm{W}=\frac{4 \sqrt{2}}{3 \mathrm{~g}^{2}}-\left[\mathrm{n}+\frac{1}{2}\right]-\left[\mathrm{n}+\frac{1}{2}\right] \ln \left[\frac{2^{9 / 2}}{\mathrm{~g}^{2}\left(\mathrm{n}+\frac{1}{2}\right)}\right]
$$

Inserting this into (5.7) (where in the domain under discussion $k \simeq 1$ and $\mathcal{X}\left(\gamma^{\prime}\right) \simeq X(0)$ $\left.=\frac{1}{2} \pi\right)$ we obtain

$$
\operatorname{Im} E=\frac{\mu}{\pi \sqrt{2}} e^{-\frac{4 \sqrt{2}}{3 g^{2}}} e^{n+\frac{1}{2}} \cdot\left[\frac{16 \sqrt{2}}{g^{2}\left(n+\frac{1}{2}\right)}\right]^{n+\frac{1}{2}}
$$

The exponential suppression factor is seen to be identical with that for vacuum bounces 3 ). An interesting observation is that for the case of weak coupling under discussion the tunneling effect described by (5.8) indeed grows with energy (i.e. $n$ ) exponentially, of course in the domain of validity of the expansions used in deriving (5.8), i.e. for

$$
2 \sqrt{2} \mathrm{~g}^{2}\left[\mathrm{n}+\frac{1}{2}\right]=\mathrm{u}<<1
$$

This observation is in agreement with common belief ${ }^{17)}$ in the analysis of baryon- and lepton-number violation at high energies or high temperature in models possessing instantons. Using Stirling's formula in the form

$$
\left[\frac{\mathrm{e}}{\mathrm{n}+\frac{1}{2}}\right]^{\mathrm{n}+\frac{1}{2}} \simeq \frac{\sqrt{2 \pi}}{\mathrm{n} !}
$$

(obtained with the help of $\exp z=\lim _{n \rightarrow \infty}\left[n+\frac{z}{n}\right]^{n}$ ) we can rewrite (5.8) as

$$
\operatorname{Im} E=\frac{\mu}{\sqrt{\pi} n !}\left[\frac{16 \sqrt{2}}{g^{2}}\right]^{n+\frac{1}{2}} e^{-\frac{4 \sqrt{2}}{3 g^{2}}}
$$


This result agrees with the complex energy eigenvalue of the Schrödinger equation for the inverted double-well potential obtained by Bender and $\mathrm{Wu}^{18)}$ using a WKB analysis and by others ${ }^{19)}$ with an alternative method. It also reduces to the result obtained from a calculation with vacuum bounces for the metastable ground state ${ }^{3}$ ). (In the comparison with the literature, e.g. ref. [19], it must be remembered that here we take the mass of the particle to be one, whereas in quantum mechanics calculations one frequently takes one half; thus in the comparison with ref. [19] $\mathrm{E}$ there is $2 \mathrm{E}$ here, $\mathrm{h}^{2}$ there is $2 \sqrt{2} \mu$ here, and $\mathrm{C}^{2}$ is $2 \mu^{2} / \mathrm{a}^{2}$ here).

(B) If the energy approaches the barrier height, i.e. $\mathrm{E} \rightarrow \frac{1}{2} \mathrm{a}^{2} \mu^{2}$ with $\mathrm{k}$ or $\gamma \rightarrow 0$, the complete elliptic integrals $\mathrm{E}(\gamma)$ and $\chi(\gamma)$ in (4.23) have to be expressed as power series in ascending powers of $\gamma$, i.e.

$$
\begin{aligned}
& \mathrm{E}(\mathcal{X}(\gamma))=\frac{\pi}{2}\left[1-\frac{1}{4} \gamma^{2}+\ldots\right] \\
& \chi(\gamma)=\frac{\pi}{2}\left[1+\frac{1}{4} \gamma^{2}+\ldots\right]
\end{aligned}
$$

and the argument of the exponential in (4.23) is

$$
-\mathrm{W}=0 \text { with } \gamma^{2} \rightarrow 0
$$

Looking at (4.22) one might conclude that the transition amplitude is then not suppressed by the typical vacuum tunneling factor $\exp \left[-\frac{4 \sqrt{2}}{3 g^{2}}\right]$. However, the prefactor in (4.23), i.e.

$$
\frac{\mu(1+\mathrm{k})}{4 \sqrt{1+\mathrm{k}^{2}} \chi\left(\gamma^{\prime}\right)} \simeq \frac{\mu}{4 \ln \left(\frac{4}{\gamma}\right)}
$$

also approaches zero as $\mathbf{k}$ or $\gamma \rightarrow 0$, and thus the transition amplitude is again suppressed (though not by the vacuum tunneling factor). This phenomenon might appear as a new observation for quantum tunneling at high energy (where, one could think naively, the 
tunneling is not suppressed), but this interpretation originates obviously from a semiclassical point of view. When the energy is very high the effect of anharmonic oscillations becomes important for the inverted double-well potential, and the effective frequency, namely the number of impacts per unit time at the turning points approaches zero.

\section{The Bogomolny-Fateyev relation}

In discussions of the large-order behaviour of perturbation theory in gauge theories Bogomolny and Fateyev ${ }^{20}$ ) estimated the behaviour of the coefficients of the perturbation expansion around a nonunique vacuum state. It is pointed out that in general the classical vacuum state does not coincide with the true quantum mechanical ground state. Therefore although the exact ground state is stable, the perturbation theory vacuum is only a metastable one due to the possibility of tunneling to the other vacuum state. If one considers perturbation theory around a local minimum, the perturbation theory is metastable. The imaginary part of the energy of the metastable ground state can be calculated by the method of steepest descent. For the double-well potential

$$
\mathrm{V}(\phi)=\frac{1}{2} \lambda^{2}\left[\phi^{2}-\frac{1}{\lambda^{2}}\right]^{2}
$$

Bogomolny and Fateyev ${ }^{21)}$ find the relation

$$
\Delta \mathrm{E}=2 \pi \mathrm{i}(\delta \mathrm{E})^{2}
$$

where $\Delta \mathrm{E}$ is the discontinuity of the ground state energy at the cut $\lambda^{2} \geq 0$, while $\delta \mathrm{E}$ is the instanton contribution to the real part of the ground state energy, namely, the level shift due to quantum tunneling ${ }^{8}$. The relation (6.2) has been reconsidered ${ }^{19)}$ recently by solving Schrödinger-equations using modified WKB methods. Since there is no real decay in the system, the calculated imaginary part is the probability of tunneling away from one minimum only. Therefore the potential is considered in two forms in ref. [19]. The first form considered, $(6.1)$, leads to the level splitting $\delta \mathrm{E}$, while the second, shifted form 


$$
V(\phi)=\left\{\begin{array}{cc}
\frac{1}{2} \lambda^{2}\left(\phi^{2}-\frac{1}{\lambda^{2}}\right)^{2} \text { for } \phi \leq \frac{1}{\lambda} \\
-\frac{1}{2} \lambda^{2}\left(\phi^{2}-\frac{1}{\lambda^{2}}\right)^{2} & \text { for } \phi>\frac{1}{\lambda}
\end{array}\right.
$$

as shown in Fig. 2, results in the imaginary part of the energy, Im E, for a "real" metastable ground state. Since $\Delta \mathrm{E}=2 \mathrm{i}$ Im $\mathrm{E}$, one has the equivalent relation

$$
\operatorname{Im} \mathrm{E}=\pi(\delta \mathrm{E})^{2}
$$

which has been verified and extended to excited states (low energy case) in ref. [20] by comparing $\operatorname{Im} \mathrm{E}$ and $\delta \mathrm{E}$ for the two systems.

Formula (6.4) serves as a crucial test of the validity of calculating quantum tunneling effects with nonvacuum instantons ${ }^{8)}$ and bounces. In our earlier paper ${ }^{8}$ ) the level splitting for the excited states of the potential (6.1) was obtained with nonvacuum instantons which we also called periodic instantons. The classical solution which extremizes the Euclidean action is ${ }^{11)}$

$$
\phi_{\mathrm{C}}(\tau)=\frac{\mathbf{k b}(\mathbf{k})}{\lambda} \mathrm{sn}\left[\mathrm{b}(\mathbf{k})\left(\tau+\tau_{0}\right) \mid \mathbf{k}\right]
$$

where

$$
\mathbf{k}^{2}=\frac{1-\mathrm{u}}{1+\mathrm{u}}, \mathrm{u}=\lambda \sqrt{2 \mathrm{E}}, \mathrm{b}(\mathbf{k})=\left[\frac{2}{1+\mathrm{k}^{2}}\right]^{\frac{1}{2}}
$$

The Jacobian elliptic function sn $[\mathbf{z} \mid \mathbf{k}]$ has period

$$
\tau=4 \mathrm{n} X(\mathrm{k})
$$

The small fluctuation equation about $\phi_{c}(\tau)$ of $(6.5)$ is also a Lamé equation and the eigenmodes are the same as for nonvacuum bounces (c.f. Section 2) but with different eigenvalues $^{11}$ ). In the calculation of the level splitting the solution for a half period is regarded as an instanton configuration, whereas the solution for a full period is a pair of instanton 
and antiinstanton configurations. The trajectory (for $\mathrm{n}=1$ and $\tau_{0}=0$ in eq. (6.5)) of one nonvacuum instanton (or periodic instanton in ref. [8]) is indicated by the solid line in Fig. 2. The level shift (i.e. half of the level splitting defined in ref. [8]) is obtained a

$$
\delta \mathrm{E}=\mathrm{B} \exp \left[-\mathrm{W}^{\prime}\right]
$$

where the prefactor $B$ is given by

$$
\mathrm{B}=\frac{[1+\mathrm{u}]^{\frac{1}{2}}}{2 \mathcal{K}\left(\mathrm{k}^{\prime}\right)}
$$

and $\mathrm{W}^{\prime}$ by

$$
\mathrm{W}^{\prime}=\frac{4}{3 \lambda^{2}}(1+\mathrm{u})^{\frac{1}{2}}[\mathrm{E}(\mathrm{k})-\mathrm{u} X(\mathrm{k})]
$$

If we regard the configuration over the full period as a bounce configuration which returns to its original position as indicated by the dotted line in Fig. 2 (for $\mathrm{n}=1$ and $\tau_{0}=0$ ) we can write it

$$
\oint_{\mathrm{c}}(\tau)=\frac{\mathrm{kb}(\mathrm{k})}{\lambda} \mathrm{sn}\left[\mathrm{b}(\mathrm{k})\left(\tau+\tau_{0}\right)+\mathcal{X}(\mathbf{k})\right]
$$

This motion is allowed for a physical system with potential (6.3). The imaginary part of the energy is obtained the way we obtained it in this paper. We then have

$$
\operatorname{Im} \mathrm{E}=\mathrm{B} \exp \left(-2 \mathrm{~W}^{\prime}\right)
$$

and so

$$
\operatorname{Im} \mathrm{E}=\frac{1}{\mathrm{~B}}(\delta \mathrm{E})^{2}
$$

In the low energy limit $\frac{1}{B}=\pi$, and the Bogmolny-Fateyev relation holds exactly. 


\section{Acknowledgement}

One of us (J.-Q.L.) is indebted to the German Academic Exchange Service (DAAD) for financial support and the Department of Physics, University of Kaiserslautern, for its hospitality.

\section{Appendix A}

Here we show that of the three discrete fluctuation modes with negative eigenvalues, only one contributes to the tunneling.

The five discrete eigenmodes $\psi_{i}$ of the fluctuation equation and their respective eigenvalues are given by $(2.7 \mathrm{a})$ and $(2.7 \mathrm{~b})$. The boundary conditions which the fluctuation field $\mathcal{X}$ has to satisfy are

$$
\chi(\tau= \pm \mathrm{T})=0 \quad \text { or } \chi(\mathrm{z}= \pm \mathcal{X}(\gamma))=0
$$

We set

$$
\chi=\stackrel{\mathrm{n}=1}{\Sigma}_{\mathrm{n}} \mathrm{C}_{\mathrm{n}}
$$

Using $\operatorname{sn}[-\mathrm{u} \mid \gamma]=-\operatorname{sn}[\mathrm{u} \mid \gamma], \operatorname{sn}[\mathcal{X}(\gamma) \mid \gamma]=1, \operatorname{cn}[\mathcal{X}(\gamma) \mid \gamma]=0$ and $\operatorname{dn}[\mathcal{K}(\gamma) \mid \gamma]=\gamma^{\prime}$ the two conditions (A.1) imply

$$
\begin{aligned}
& -C_{2} \gamma^{\prime}+C_{4}\left[1-\frac{\Delta_{1}+\Delta_{2}}{3 \gamma^{2}}\right]+C_{5}\left[1-\frac{\Delta_{1}-\Delta_{2}}{3 \gamma^{2}}\right]=0 \\
& +C_{2} \gamma^{\prime}+C_{4}\left[1-\frac{\Delta_{1}+\Delta_{2}}{3 \gamma^{2}}\right]+C_{5}\left[1-\frac{\Delta_{1}-\Delta_{2}}{3 \gamma^{2}}\right]=0
\end{aligned}
$$

where

$$
\Delta_{1}=1+\gamma^{2}, \quad \Delta_{2}=\sqrt{1-\gamma^{2} \gamma^{\prime 2}}
$$


These conditions immediately imply $\mathrm{C}_{2}=0$ and

$$
\mathrm{C}_{4}\left[1-\frac{\Delta_{1}+\Delta_{2}}{3 \gamma^{2}}\right]=-\mathrm{C}_{5}\left[1-\frac{\Delta_{1}-\Delta_{2}}{3 \gamma^{2}}\right]
$$

Now

$$
\begin{aligned}
\mathrm{C}_{4}= & \int \chi \psi_{4} \mathrm{dz} \\
& =\int_{-\chi}^{+\chi} \chi \mathrm{sn}^{2}[\mathrm{z} \mid \gamma] \mathrm{dz}-\frac{\Delta_{1}+\Delta_{2}}{3 \gamma^{2}} \int_{-\chi}^{+\chi} \chi \mathrm{dz} \\
& =\int_{-\chi}^{+\chi} \chi \operatorname{sn}^{2}[\mathrm{z} \mid \gamma] \mathrm{dz}
\end{aligned}
$$

since $\int_{-\chi}^{+\chi} \chi \mathrm{d} z=0$ as a consequence of the periodicity of $\chi$. Similarly $\mathrm{C}_{5}$ is found to be equal to the same result (A.5). Thus $\mathrm{C}_{4}=\mathrm{C}_{5}$. From (A.4) we therefore conclude that

$$
\mathrm{C}_{4}=\mathrm{C}_{5}=0
$$

Thus only $\psi_{1}$ with $\mathrm{E}_{1}=0$ and $\psi_{3}$ with $\mathrm{E}_{3}=-3 \mu^{2}(1+\mathrm{k})^{2} /\left(1+\mathrm{k}^{2}\right)$ contribute to the tunneling.

\section{Appendix B}

Here we evaluate the functional integral I defined by (4.3b) and derive the result (4.10).

From (4.2) and (4.9) we see that

$$
\begin{aligned}
& \mathrm{y}\left(\tau_{\mathrm{i}}\right)=0 \\
& \mathrm{y}\left(\tau_{\mathrm{f}}\right)+\mathrm{N}\left(\tau_{\mathrm{f}}\right) \int_{\tau_{\mathrm{i}}}^{\tau_{\mathrm{f}}} \frac{\dot{\mathrm{N}}\left(\tau^{\prime}\right)}{\mathrm{N}^{2}\left(\tau^{\prime}\right)} \mathrm{y}\left(\tau^{\prime}\right) \mathrm{d} \tau^{\prime}=0
\end{aligned}
$$


Substitution of (4.9) into (4.5) yields

$$
\begin{aligned}
& \delta S=\frac{1}{2} \int_{\tau_{\mathrm{i}}}^{\tau_{\mathrm{f}}} \mathrm{d} \tau \chi \hat{\mathrm{M}} \chi \\
& =\frac{1}{2} \int_{\tau_{\dot{i}}}^{\tau_{\mathrm{f}}} \mathrm{d} \tau\left\{\mathrm{y}+\mathrm{N} \int_{\tau_{\mathrm{i}}}^{\tau} \frac{\mathbf{N}\left(\tau^{\prime}\right)}{\mathrm{N}^{2}\left(\tau^{\prime}\right)} \mathrm{y}\left(\tau^{\prime}\right) \mathrm{d} \tau^{\prime}\right\} \hat{\mathbf{M}} \\
& \cdot\left\{\mathrm{y}+\mathrm{N} \int_{\tau_{\mathrm{i}}}^{\tau} \frac{\mathrm{N}\left(\tau^{\prime \prime}\right)}{\mathrm{N}^{2}\left(\tau^{\prime \prime}\right)} \mathrm{y}\left(\tau^{\prime \prime}\right) \mathrm{d} \tau^{\prime \prime}\right\}
\end{aligned}
$$

Using

$$
\hat{\mathrm{M}}:=-\frac{\mathrm{d}^{2}}{\mathrm{~d} \tau^{2}}+\mathrm{g}(\tau), \mathrm{g}(\tau)=2\left(\mu^{2}-\frac{3 \mu^{2}}{\mathrm{a}^{2}} \phi_{\mathrm{c}}^{2}\right)
$$

and

$$
\hat{\mathrm{MN}}=0, \ddot{\mathrm{N}}=\mathrm{gN}
$$

and partial integrations, and dropping total derivative contributions which vanish because $\mathrm{N}\left(\tau_{\mathrm{i}}\right)=0=\mathrm{N}\left(\tau_{\mathrm{f}}\right)$, we have

$$
\begin{aligned}
& \delta S=\frac{1}{2} \int_{\tau_{i}}^{\tau_{f}}\left\{y+N \int \ldots\right\}\left[-\ddot{y}+g y-\frac{2 \hat{N}^{2}}{N^{2}} y-N \frac{d}{d \tau}\left[\frac{N y}{N^{2}}\right]\right] \\
& =\frac{1}{2} \int_{\tau_{i}}^{\tau_{\mathrm{f}}} \mathrm{d} \tau\left[-\mathrm{y} \ddot{\mathrm{y}}-\ddot{\mathrm{y} N} \int \ldots+\mathrm{y}^{2} \mathrm{~g}+\mathrm{Ngy} \int \ldots-\frac{\dot{\mathrm{N}}^{2} \mathrm{y}^{2}}{\mathrm{~N}^{2}}-\frac{\mathrm{d}}{\mathrm{d} \tau}\left(\mathrm{Ny} \int \ldots\right)\right. \\
& \left.-\mathrm{yN} \frac{\mathrm{d}}{\mathrm{d} \tau}\left[\frac{\mathrm{Ny}}{\mathrm{N}^{2}}\right]\right] \\
& =\frac{1}{2} \int_{\tau_{i}}^{\tau_{f}} \mathrm{~d} \tau\left[-\mathrm{y} \ddot{\mathrm{y}}-\dot{\mathrm{y}}\left\{\dot{\mathrm{N}} \int \ldots+\frac{\grave{N} \mathrm{y}}{\mathrm{N}}\right\}+\mathrm{y}^{2} \mathrm{~g}+\mathrm{Ngy} \int \ldots-\frac{\dot{\mathrm{N}}^{2} \mathrm{y}^{2}}{\mathrm{~N}^{2}}\right. \\
& \left.-\mathrm{y}^{2} \mathrm{~N} \frac{\mathrm{d}}{\mathrm{d} \tau}\left[\frac{\hat{\mathrm{N}}}{\mathrm{N}^{2}}\right]-\mathrm{y} \frac{\mathrm{N}}{\mathrm{N}} \dot{\mathrm{y}}\right]
\end{aligned}
$$




$$
\begin{aligned}
& =\frac{1}{2} \int_{\tau_{\mathrm{i}}}^{\tau_{\mathrm{f}}} \mathrm{d} \tau\left[-\mathrm{y} \ddot{\mathrm{y}}-\mathrm{y} \ddot{\mathrm{N}} \int \ldots-\mathrm{y}^{2} \frac{\dot{\mathrm{N}}^{2}}{\mathrm{~N}^{2}}+\mathrm{y}^{2} \mathrm{~g}+\mathrm{Ngy} \int \ldots-\frac{\dot{\mathrm{N}}^{2}}{\mathrm{~N}^{2}} \mathrm{y}^{2}-\mathrm{y}^{2} \mathrm{~N} \frac{\mathrm{d}}{\mathrm{d} \tau}\left[\frac{\mathrm{N}}{\mathrm{N}^{2}}\right]\right] \\
& =\frac{1}{2} \int_{\tau_{\mathrm{i}}}^{\tau_{\mathrm{f}}} \mathrm{d} \tau\left[-\mathrm{y} \ddot{\mathrm{y}}-\mathrm{y}^{2}\left\{2 \frac{\mathrm{N}^{2}}{\mathrm{~N}^{2}}-\frac{\ddot{\mathrm{N}}}{\mathrm{N}}+\mathrm{N} \frac{\mathrm{d}}{\mathrm{d} \tau}\left[\frac{\mathrm{N}}{\mathrm{N}^{2}}\right]\right\}\right] \\
& =\frac{1}{2} \int_{\tau_{\mathrm{i}}}^{\tau_{\mathrm{f}}} \mathrm{d} \tau[\mathrm{y} \ddot{\mathrm{y}}] \\
& =\frac{1}{2} \int_{\tau_{\mathrm{i}}}^{\tau_{\mathrm{f}}} \mathrm{d} \tau\left[\dot{\mathrm{y}}^{2}\right]
\end{aligned}
$$

Thus the expression reduces to that of a free particle propagator subject to the constraints (B.1). We insert (B.5) into I defined by (4.3b) with the help of the following identity:

$$
\begin{aligned}
1=\int \mathrm{dy}_{\mathrm{f}} \delta\left(\mathrm{y}_{\mathrm{f}}+\mathrm{f}\left(\tau_{\mathrm{f}}\right)\right) \\
\quad=\int \mathrm{dy}_{\mathrm{f}} \frac{1}{2 \pi} \int \mathrm{d} \alpha \exp \left[-\mathrm{i} \alpha\left(\mathrm{y}_{\mathrm{f}}+\mathrm{f}\left(\tau_{\mathrm{f}}\right)\right)\right]
\end{aligned}
$$

where

$$
\mathrm{y}_{\mathrm{f}} \equiv \mathrm{y}\left(\tau_{\mathrm{f}}\right) \text { and } \mathrm{f}\left(\tau_{\mathrm{f}}\right) \equiv \mathrm{N}\left(\tau_{\mathrm{f}}\right) \int_{\tau_{\mathrm{i}}}^{\tau_{\mathrm{f}}} \frac{\dot{N}\left(\tau^{\prime}\right)}{N^{2}\left(\tau^{\prime}\right)} \mathrm{y}\left(\tau^{\prime}\right) \mathrm{d} \tau^{\prime}
$$

With a partial integration we can write

$$
\mathrm{y}_{\mathbf{f}}+\mathrm{f}\left(\tau_{\mathrm{f}}\right)=\mathrm{N}\left(\tau_{\mathrm{f}}\right) \int_{\tau_{\mathrm{i}}}^{\tau_{\mathrm{f}}} \frac{1}{\mathrm{~N}(\tau)} \frac{\mathrm{dy}}{\mathrm{d} \tau} \mathrm{d} \tau
$$

We can therefore write I in the form 


$$
\begin{aligned}
& \mathrm{I}=\frac{\mathrm{I}}{2 \pi} \int \mathrm{dy}_{\mathrm{f}} \int_{\mathrm{y}\left(\tau_{\mathrm{i}}\right)=0}^{\mathrm{y}\left(\tau_{\mathrm{f}}\right)} \mathcal{D}\{\mathrm{y}\}\left|\frac{\partial \chi}{\partial \mathrm{y}}\right| \cdot \int_{-\infty}^{\infty} \mathrm{d} \alpha \\
& \cdot \exp \left[-\int_{\tau_{\mathbf{i}}}^{\tau_{\mathrm{f}}} \frac{1}{2}\left[\frac{\mathrm{dy}}{\mathrm{d} \tau}\right]^{2} \mathrm{~d} \tau\right] \\
& \cdot \exp \left[-\mathrm{i} \alpha\left(\mathrm{y}\left(\tau_{\mathrm{f}}\right)+\mathrm{f}\left(\tau_{\mathrm{f}}\right)\right)\right] \\
& =\int \frac{\mathrm{dy}_{\mathrm{f}}}{2 \pi}\left|\frac{\partial \chi}{\partial \mathrm{y}}\right| \int_{\mathrm{y}\left(\tau_{\mathrm{i}}\right)=0}^{\mathrm{y}\left(\tau_{\mathrm{f}}\right)} \mathcal{D}\{\mathrm{y}\} \\
& \cdot \exp \left[-\frac{1}{2} \int_{\tau_{\mathrm{i}}}^{\tau_{\mathrm{f}}}\left\{\frac{\mathrm{dy}}{\mathrm{d} \tau}+\mathrm{i} \alpha \frac{\mathrm{N}\left(\tau_{\mathrm{f}}\right)}{\mathrm{N}(\tau)}\right\}^{2} \mathrm{~d} \tau\right] \\
& \cdot \exp \left[-\frac{\alpha^{2}}{2} \mathrm{~N}^{2}\left(\tau_{\mathrm{f}}\right) \int_{\tau_{\mathrm{i}}}^{\tau_{\mathrm{f}}} \frac{\mathrm{d} \tau}{\mathrm{N}^{2}(\tau)}\right] \mathrm{d} \alpha
\end{aligned}
$$

We can write this

$$
\begin{array}{r}
\mathrm{I}=\int \frac{\mathrm{dy}_{\mathrm{f}}}{2 \pi}\left|\frac{\partial \chi}{\partial \mathrm{y}}\right| \boldsymbol{x}_{0}\left(\mathrm{y}_{\mathrm{f}}, \tau_{\mathrm{f}}, \mathrm{y}\left(\tau_{\mathrm{i}}\right)=0, \tau_{\mathrm{j}}\right) \\
\quad \cdot \exp \left[-\frac{\alpha^{2}}{2} \mathrm{~N}^{2}\left(\tau_{\mathrm{f}}\right) \int_{\tau_{\mathrm{i}}}^{\tau_{\mathrm{f}}} \frac{\mathrm{d} \tau}{\mathrm{N}^{2}(\tau)}\right] \mathrm{d} \alpha
\end{array}
$$

where $x_{0}$ is the configuration space representation of the free nonrelativistic Green's function with

$$
\int x_{0}\left(y_{f}, \tau_{f} ; y\left(\tau_{i}\right)=0, \tau_{i}\right) d y_{f}=1
$$


Hence

$$
\mathrm{I}=\frac{1}{\sqrt{2 \pi}}\left|\frac{\partial \chi}{\partial \mathrm{y}}\right|\left[\mathrm{N}^{2}\left(\tau_{\mathrm{f}}\right) \int_{\tau_{\mathrm{i}}}^{\tau_{\mathrm{f}}} \frac{\mathrm{d} \tau}{\mathrm{N}^{2}(\tau)}\right]^{-\frac{1}{2}}
$$

In the literature ${ }^{14)}$ it is shown that the Jacobian of the transformation (4.9) is given by

$$
\left|\frac{\partial \chi}{\partial y}\right|=\sqrt{\frac{N\left(\tau_{f}\right)}{N\left(\tau_{i}\right)}}
$$

Thus

$$
\mathrm{I}=\frac{1}{\sqrt{2 \pi}}\left[\mathrm{N}\left(\tau_{\mathrm{i}}\right) \mathrm{N}\left(\tau_{\mathrm{f}}\right) \int_{\tau_{\mathrm{i}}}^{\tau_{\mathrm{f}}} \frac{\mathrm{d} \tau}{\mathrm{N}^{2}(\tau)}\right]^{-\frac{1}{2}}
$$

This establishes $(4.10)$ of the text.

\section{Appendix C}

Here we establish the relations (4.11b) and (4.12b).

Our derivation is based on the following formula which we therefore prove first, i.e.

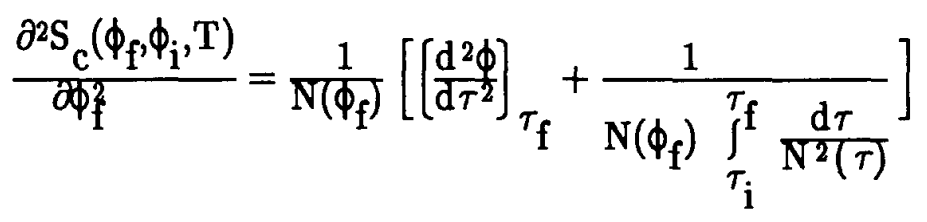

For $\tau_{\mathrm{j}}, \tau_{\mathrm{f}}=\mp \mathrm{T} \rightarrow \mp \infty$ the corresponding formula was derived in the Appendix ref. [8].

In demonstrating (C.1) we start from

$$
\mathrm{S}\left(\phi_{\mathrm{f}}, \phi_{\mathrm{i}} ; \mathrm{T}\right)=\mathrm{W}\left(\phi_{\mathrm{f}}, \phi_{\mathrm{i}} ; \mathrm{E}\right)+2 \mathrm{ET}
$$


where

$$
E \equiv E\left(\phi_{f}, \phi_{i} ; T\right)
$$

From (C.2) we obtain

$$
0=\frac{\partial \mathrm{S}}{\partial \mathrm{E}}=\frac{\partial \mathrm{W}}{\partial \mathrm{E}}+2 \mathrm{~T}
$$

and

$$
\begin{aligned}
\frac{\partial \mathrm{S}}{\partial \phi_{\mathrm{f}}}= & \frac{\partial \mathrm{W}}{\partial \phi_{\mathrm{f}}}+\frac{\partial \mathrm{W}}{\partial \mathrm{E}} \frac{\partial \mathrm{E}}{\partial \phi_{\mathrm{f}}}+2 \mathrm{~T} \frac{\partial \mathrm{E}}{\partial \phi_{\mathrm{f}}} \\
& =\frac{\partial \mathrm{W}\left(\phi_{\mathrm{f}}, \phi_{\mathrm{i}} ; \mathrm{E}\right)}{\partial \phi_{\mathrm{f}}}
\end{aligned}
$$

with the help of (C.4). Further,

$$
\frac{\partial^{2} \mathrm{~S}}{\partial \phi_{\mathrm{f}}^{2}}=\frac{\partial^{2} \mathrm{~W}}{\partial \phi_{\mathrm{f}}}+\frac{\partial^{2} \mathrm{~W}}{\partial \mathrm{E} \partial \phi_{\mathrm{f}}} \frac{\partial \mathrm{E}}{\partial \phi_{\mathrm{f}}}
$$

Now from (C.2) we see that

$$
E=\frac{\partial S}{\partial(2 T)}
$$

so that with (C.5)

$$
\begin{aligned}
\frac{\partial \mathrm{E}}{\partial \phi_{\mathrm{f}}}= & \frac{\partial}{\partial \phi_{\mathrm{f}}}\left[\frac{\partial \mathrm{S}}{\partial(2 \mathrm{~T})}\right] \\
& =\frac{\partial}{\partial(2 \mathrm{~T})}\left[\frac{\partial \mathrm{S}}{\partial \phi_{\mathrm{f}}}\right] \\
& =\frac{\partial}{\partial(2 \mathrm{~T})}\left[\frac{\partial \mathrm{W}}{\partial \phi_{\mathrm{f}}}\right]
\end{aligned}
$$




$$
\begin{aligned}
& =\frac{\partial}{\partial \mathrm{E}}\left[\frac{\partial \mathrm{W}}{\partial \phi_{\mathrm{f}}}\right] \frac{\partial \mathrm{E}}{\partial(2 \mathrm{~T})} \\
& =\frac{\partial^{2} \mathrm{~W}}{\partial \mathrm{E} \partial \phi_{\mathrm{f}}} \frac{1}{\partial(2 \mathrm{~T})} \frac{\partial \mathrm{E}}{\partial{ }^{2}}
\end{aligned}
$$

Using (C.4) we can rewrite this

$$
\frac{\partial \mathrm{E}}{\partial \Phi_{\mathrm{f}}}=-\frac{\partial^{2} \mathrm{~W}}{\partial \mathrm{E} \partial \phi_{\mathrm{f}}} / \frac{\partial^{2} \mathrm{~W}}{\partial \mathrm{E}^{2}}
$$

Inserting this into (C.6) we obtain

$$
\frac{\partial^{2} S}{\partial \phi_{\mathrm{f}}^{2}}=\frac{\partial^{2} \mathrm{~W}}{\partial \phi_{\mathrm{f}}^{2}}-\left[\frac{\partial^{2} \mathrm{~W}}{\partial \phi_{\mathrm{f}} \partial \mathrm{E}}\right]^{2} / \frac{\partial^{2} \mathrm{~W}}{\partial \mathrm{E}^{2}}
$$

Now

$$
\mathrm{W}=\int_{\tau_{\mathrm{i}}}^{\tau_{\mathrm{f}}}\left[\frac{\mathrm{d} \phi}{\mathrm{d} \tau}\right]^{2} \mathrm{~d} \tau=\oint_{\oint_{\mathrm{i}}}^{\phi_{\mathrm{f}}} \mathrm{d} \phi \frac{\mathrm{d} \phi}{\mathrm{d} \tau}
$$

so that

$$
\frac{\partial \mathrm{W}}{\partial \phi_{\mathrm{f}}}=(\dot{\phi})_{\phi_{\mathrm{f}}}=\left.\sqrt{2(\nabla-\mathrm{E})}\right|_{\phi_{\mathrm{f}}}
$$

It follows that

$$
\begin{aligned}
& \frac{\partial^{2} \mathrm{~W}}{\partial \phi_{\mathrm{f}} \partial \mathrm{E}}=-\left[\frac{1}{\dot{\phi}}\right]_{\phi_{\mathrm{f}}} \\
& \frac{\partial^{2} \mathrm{~W}}{\partial \mathrm{E}^{2}}=-\int_{\tau_{\mathrm{i}}}^{\tau_{\mathrm{f}}} \frac{\mathrm{d} \tau}{2} \\
& \frac{\partial^{2} \mathrm{~W}}{\partial \phi_{\mathrm{f}}}=\frac{\left[\frac{\partial \mathrm{V}}{\partial \phi}\right]_{\phi_{\mathrm{f}}}}{\sqrt{2\left(\mathrm{~V}\left(\phi_{\mathrm{f}}\right)-\mathrm{E}\right)}}=\left[\begin{array}{l}
\Phi \\
\dot{\phi}
\end{array}\right]_{\phi_{\mathrm{f}}}
\end{aligned}
$$


where in the last step we used the equation of motion. Inserting (C.12), (C.13) and (C.14) into (C.9) and setting $\dot{\phi}=\mathrm{N}(\tau), \phi_{\mathrm{f}}=\phi\left(\tau_{\mathrm{f}}\right)$, we obtain (C.1).

We now consider the relation (4.11) which we rewrite in the form

$$
\Delta=\lim _{\substack{\phi_{\mathrm{i}} \rightarrow \phi(-\mathrm{T}) \\ \phi_{\mathrm{f}} \rightarrow \phi(\mathrm{T})}}\left[1+\frac{\mathrm{d} \phi_{\mathrm{f}}}{\mathrm{d} \tau} \frac{\mathrm{d}^{2} \phi_{\mathrm{f}}}{\mathrm{d} \tau^{2}} \int_{\tau_{\mathrm{i}}}^{\tau_{\mathrm{f}}} \frac{\mathrm{d} \tau}{\mathrm{N}^{2}(\tau)}\right]^{-\frac{1}{2}}
$$

From (4.6) we obtain

$$
\begin{aligned}
& \frac{\mathrm{d} \phi_{\mathrm{C}}}{\mathrm{d} \tau}=-\mathrm{s}_{+}(\mathrm{k}) \beta(\mathrm{k}) \gamma^{2} \operatorname{sn}[\beta(\mathrm{k}) \tau \mid \gamma] \operatorname{cn}[\beta(\mathrm{k}) \tau \mid \gamma] \\
& \frac{\mathrm{d}^{2} \phi_{\mathrm{C}}}{\mathrm{d} \tau^{2}}=-\mathrm{s}_{+}(\mathrm{k}) \beta^{2}(\mathrm{k}) \gamma^{2}\left\{\mathrm{cn}^{2}[\beta(\mathrm{k}) \tau \mid \gamma]-\mathrm{sn}^{2}[\beta(\mathrm{k}) \tau \mid \gamma]\right\} \operatorname{dn}[\beta(\mathrm{k}) \tau \mid \gamma]
\end{aligned}
$$

Using formula 361.10 of ref. [12] we obtain

$$
\begin{aligned}
& \int_{\tau_{\mathrm{i}}}^{\tau_{\mathrm{f}}} \frac{\mathrm{d} \tau}{\left(\mathrm{d} \Phi_{\mathrm{c}} / \mathrm{d} \tau\right)^{2}}=\frac{1}{\mathrm{~s}_{+}^{2}(\mathrm{k}) \beta^{3}(\mathrm{k}) \gamma^{4}} \int_{\beta \tau_{\mathrm{i}}}^{\beta \tau_{\mathrm{f}}} \mathrm{duns} \mathrm{ns}^{2}[\mathrm{u} \mid \gamma] \mathrm{nc}^{2}[\mathrm{u} \mid \gamma] \\
& =\frac{1}{\mathrm{~s}_{+}^{2}(\mathrm{k}) \beta^{3}(\mathrm{k}) \gamma^{4} \gamma^{\prime 2}}\left[2{\gamma^{\prime}}^{2} \mathrm{u}-\left(1+{\gamma^{\prime}}^{2}\right) \mathrm{E}(\mathrm{u})\right. \\
& \left.+\operatorname{dn}[\mathrm{u} \mid \gamma]\left\{\operatorname{tn}[\mathrm{u} \mid \gamma]-{\gamma^{\prime}}^{2} \operatorname{cs}[\mathrm{u} \mid \gamma]\right\}\right]_{\beta \tau_{\mathrm{i}}}^{\beta \tau_{\mathrm{f}}}
\end{aligned}
$$

where $\mathrm{tn}=\mathrm{sn} / \mathrm{cn}$ and $\mathrm{cs}=\mathrm{cn} / \mathrm{sn}$. In the limits $\tau_{\mathrm{i}} \rightarrow-\mathrm{T}, \tau_{\mathrm{f}} \rightarrow+\mathrm{T}$, i.e. $\beta \tau_{\mathrm{i}} \rightarrow-\chi(\gamma)$, $\beta \tau_{\mathrm{f}}++\mathcal{X}(\gamma)$ the contribution of $\operatorname{tn}[\mathrm{u} \mid \gamma]$ diverges owing to the property $\operatorname{cn}[\mathcal{X}(\gamma) \mid \gamma]=0$. However the limit $\Delta$ is finite and since in this limit 


$$
\int_{\tau_{\mathrm{i}}}^{\tau_{\mathrm{f}}} \frac{\mathrm{d} \tau}{\left(\mathrm{d} \phi_{\mathrm{c}} / \mathrm{d} \tau\right)^{2}} \rightarrow \frac{2 \mathrm{dn}[\mathcal{X}(\gamma) \mid \gamma] \operatorname{sn}[\mathcal{X}(\gamma) \mid \gamma]}{\mathrm{s}_{+}^{2}(\mathrm{k}) \beta^{3}(\mathrm{k}) \gamma^{4} \gamma^{\prime 2} \operatorname{cn}[\mathcal{X}(\gamma) \mid \gamma]}
$$

we obtain

$$
\begin{aligned}
\Delta= & {\left[1+\mathrm{s}_{+}^{2}(\mathrm{k}) \beta^{3}(\mathrm{k}) \gamma^{4} \operatorname{sn}[\mathrm{u} \mid \gamma] \operatorname{cn}[\mathrm{u} \mid \gamma] \operatorname{dn}[\mathrm{u} \mid \gamma] .\right.} \\
& \left.\cdot \frac{\left(\mathrm{cn}^{2}[\mathrm{u} \mid \gamma]-\operatorname{sn}^{2}[\mathrm{u} \mid \gamma]\right) 2 \operatorname{sn}[\mathrm{u} \mid \gamma]}{\operatorname{cn}[\mathrm{u} \mid \gamma] \mathrm{s}_{+}^{2}(\mathrm{k}) \beta^{3}(\mathrm{k}) \gamma^{4} \gamma^{\prime 2}}\right]\left.^{-\frac{1}{2}}\right|_{\mathrm{u}=\chi(\gamma)} \\
= & {[1-2]^{-\frac{1}{2}}=-\mathrm{i} }
\end{aligned}
$$

since $\operatorname{dn}[X \mid \gamma]=\gamma^{\prime}$ and $\operatorname{sn}[X \mid \gamma]=1$. 


\section{References}

1) E. Gildener and A. Patrascioiu, Phys. Rev. D16, 423 (1977)

2) S. Coleman, Phys. Rev. D15, 2929 (1977); C. Callan, Jr. and S. Coleman, ibid D16, 1726 (1977).

3) J.Q. Liang and H.J.W. Müller-Kirsten, Phys. Rev. D45, 2963 (1992); ibid D48, 964 (1993).

4) A. Ringwald, Nucl. Phys. B330, 1 (1989); also O. Espinosa, ibid B343, 310 (1990).

5) S.Yu. Khlebnikov, V.A. Rubakov and P.G. Tinyakov, Nucl. Phys. B367, 334 (1991).

6) M.E. Shaposhnikov, Phys. Lett. B242, 493 (1990).

7) K. Funakubo, S. Otsuki, K. Tekenaga and F. Toyoda, Prog. Theor. Phys. $\underline{87}, 663$ (1992).

8) J.Q. Liang and H.J.W. Müller-Kirsten, Phys. Rev. D46, 4685 (1992).

9) S. Coleman, Nucl. Phys. $\underline{B 298}, 178$ (1988).

10) N.S. Manton and T.S. Samols, Phys. Lett. B207, 179 (1988).

11) J.Q. Liang, H.J.W. Müller-Kirsten and D.H. Tchrakian, Phys. lett. B282, 105 (1992). The suppressed elliptic modules of the solution (10b) given there is not $\mathbf{k}$, as for (10a) and (10b), but $\gamma$ with $\gamma^{2}=\frac{4 \mathrm{k}}{(1+\mathrm{k})^{2}}$.

12) P.F. Byrd and M.D. Friedman, Handbook of Elliptic integrals for Engineers and Scientists, 2nd ed. (Springer, New York, 1971), formula 361.01. One should note that the authors use $\mathrm{E}(\mathrm{u})$ and $\mathrm{E}(\gamma)$ (see pp. XIV, 11) so that $\mathrm{E}(\mathrm{u}=\infty)=$ $\mathrm{E}(\mathbf{k}=1))$.

13) R.F. Dashen, B. Hasslacher and A. Neveu, Phys. Rev. D10, 4114 (1974).

14) For more detail of the calculation consult: W. Dittrich and M. Reuter, Classical and Quantum Dynamics: From classical paths to path integrals (Springer, 1992).

15) L.D. Landau and E.M. Lifshitz, Quantum Mechanics, 3rd ed. (Pergamon, New York, 1977), pp. 169-172.

16) K. Banerjee and S.P. Bhatnagar, Phys. Rev. $\underline{D 18}, 4767$ (1978); S.K. Bhattacharya, Phys. Rev. A31, 1991 (1985). 
17) J.M. Cornwall, Phys. Lett. B243, 271 (1990), J.M. Cornwall and G. Tiktopoulos, Phys. Rev. D45, 2105 (1992); H. Goldberg, Phys. Lett. B246, 495 (1990).

18) C.M. Bender and T.T. Wu, Phys. Rev. D7, 1620 (1973).

19) P. Achuthan, H.J.W. Müller-Kirsten and A. Wiedemann, Fortschr. Phys. $\underline{38}, 77$ (1990).

20) E.B. Bogomolny and V.A. Fateyev, Phys. Lett. B71, 93 (1977). 


\section{Figure Captions}

\section{Fig. 1}

Inverted double-well potential and trajectories of bounces for $n=1$ and 2 .

\section{Fig. 2}

Motion of a single nonvacuum instanton (I) and a single nonvacuum bounce (II) in the central barrier of the double-well potential. 


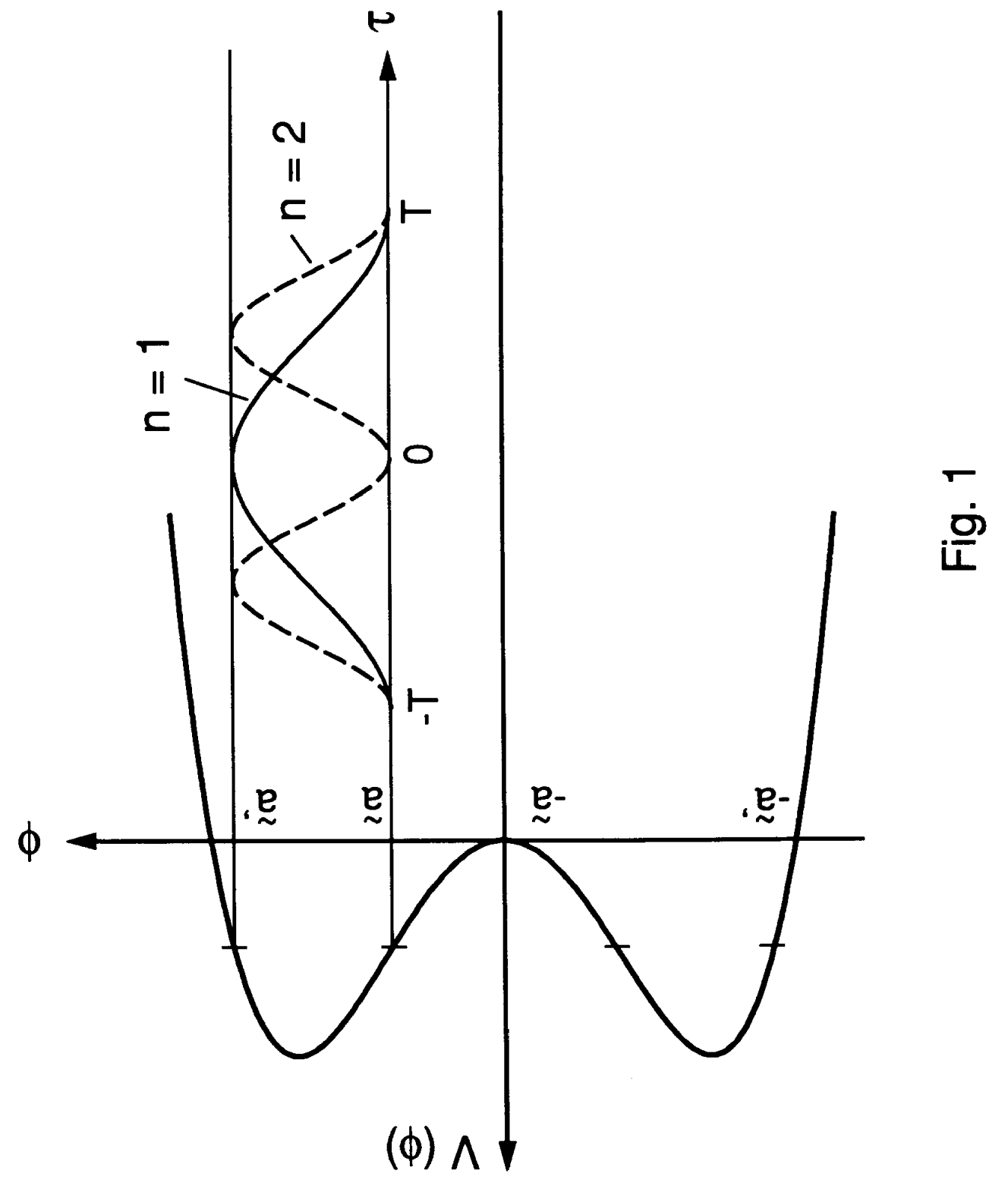




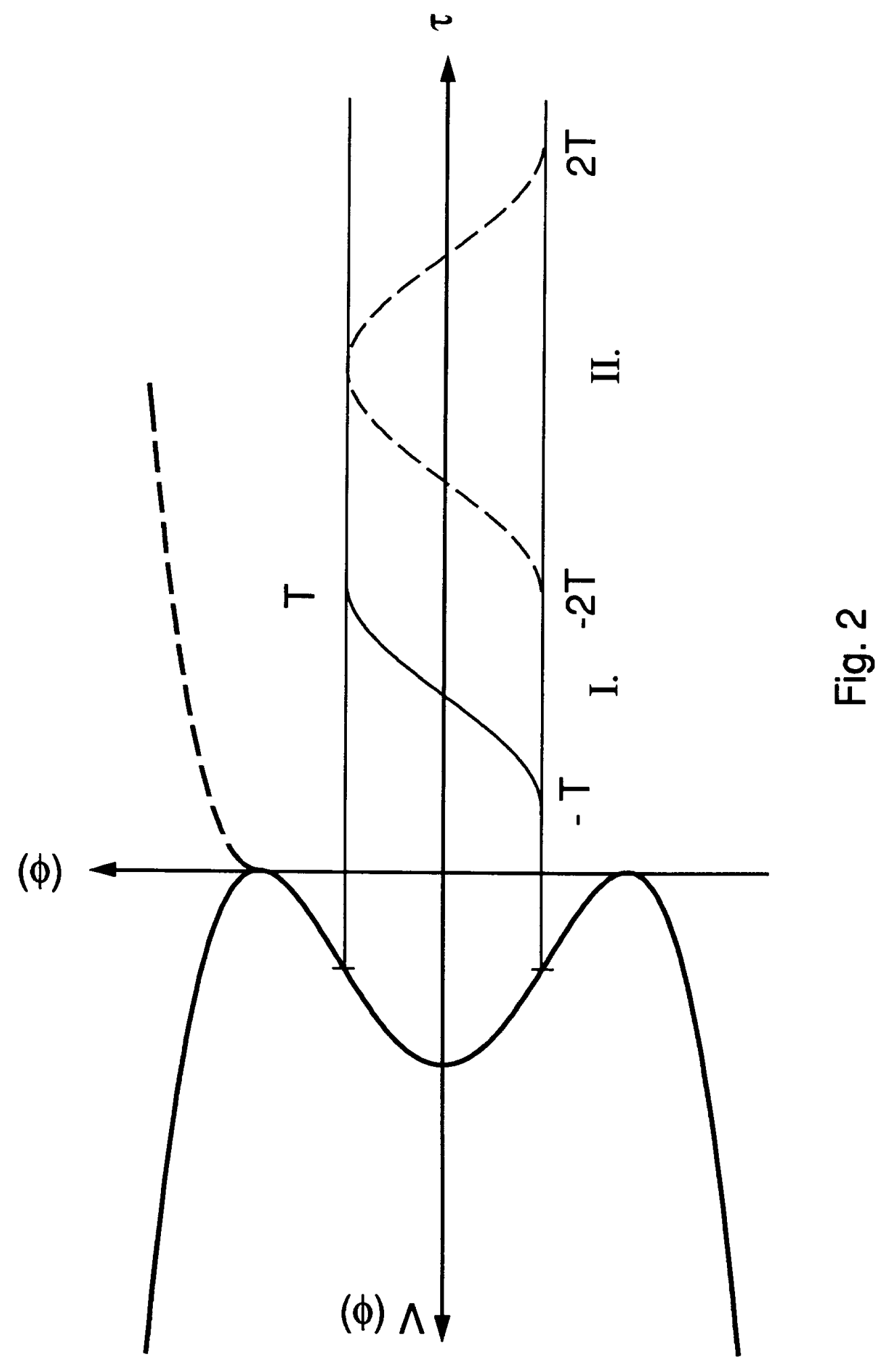

